\title{
The ATLAS transverse momentum trigger evolution at the LHC towards Run II
}

\author{
Antonia STRÜBIG* on behalf of the ATLAS collaboration \\ Radboud Universiteit, Nijmegen and Nikhef, Amsterdam (NL) \\ E-mail: antonia.struebig@cern.ch
}

\begin{abstract}
The missing transverse momentum triggers of the ATLAS experiment at the CERN Large Hadron Collider are designed to select collision events with non-interacting particles passing through the detector. Such events provide an interesting probe to search for new physics interactions beyond the Standard Model and to study Standard Model parameters such as the Higgs boson's coupling to $b$-quarks. The transverse momentum used in the trigger system is calculated from calorimeterbased global energy sums and supplemented with information from the muon detection system. The trigger operated successfully during the first running period of the Large Hadron Collider. With the start of the second running period in 2015, the Large Hadron Collider is now operating at a higher centre-of-mass energy and increased luminosity, making it more challenging to retain or improve on the Run I performance. A brief summary of the Run I performance will be presented, together with the Run II software and hardware-based improvements as well as some of the first results from the Run II data-taking period with $\sqrt{s}=13 \mathrm{TeV}$.
\end{abstract}

XXVII International Symposium on Lepton Photon Interactions at High Energies

17-22 August 2015

Ljubljana, Slovenia

${ }^{*}$ Speaker. 


\section{The missing transverse momentum trigger during Run I}

From the start of data taking at the Large Hadron Collider (LHC) in 2010 with $\sqrt{s}=7 \mathrm{TeV}$ (Run I) to the preparatory work of the data taking with $\sqrt{s}=13 \mathrm{TeV}$ (Run II) the missing transverse momentum $\left(E_{\mathrm{T}}^{\text {miss }}\right)$ trigger system underwent many changes: the steady increase in centre-of-mass energy and instantaneous luminosity forced the development of more elaborate algorithms to retain a good performance. In Run I, the ATLAS [1] trigger structure consisted of three different levels. The first level, Level 1, was a hardware based trigger, the other two levels were software based. The Level 1 output consists of the Regions of Interest (RoIs) or global event quantities which were processed by the first software based level, Level 2. At the last level, the Event Filter, the algorithms had access to the full granularity of the detector [2]. Until the end of 2011 the $E_{\mathrm{T}}^{\text {miss }}$ trigger selected events in two steps: At Level 1 the energy content of calorimeter towers are summed and translated into a value for missing transverse momentum using look-up tables. The granularity of the calorimeter towers is $\Delta \eta \times \Delta \phi=0.2 \times 0.2$ for $|\eta|<2.5$ and larger in the more forward region. If the Level 1 trigger accepted an event the Event Filter algorithm calculates the missing transverse momentum using the full calorimeter granularity of about 155000 cells [3].

In 2012 an intermediate step was added: Before running the Event Filter algorithm, the missing transverse momentum was calculated at Level 2 by summing over the output of the firmware on the Front End boards (FEB). Each FEB sums the energy content of about 128 calorimeter cells. After passing the Level 2 trigger, events were then analyzed by the Event Filter algorithm. At Event Filter level a new algorithm was added which uses topological information instead of the bare sum of the energy content of cells: The calorimeter is clustered up by selecting seed cells with an energy deposit of $|E|>4 \sigma$ and adjoint cells with $|E|>2 \sigma$, where $\sigma$ is the estimated noise level. Immediate neighbours of the seed cells are also included into the topological object independent of their energy content. The energy content of the cluster is calibrated using so-called local weights, which assign a cluster moment dependent calibration for hadronic particles. This last step could also be dropped what resulted in a slight degradation in performance.

For Run II the trigger and data acquisition system received a general upgrade. The upgrade includes the merging of the Event Filter and Level 2 into a unified high level trigger (HLT) [4]. The subsequent upgrade of algorithms is described in Section 2.

\subsection{The missing transverse momentum significance trigger algorithms}

The missing transverse momentum significance (XS) is defined as $\mathrm{XS}=E_{\mathrm{T}}^{\text {miss }} / \sigma$. Where $\sigma$ is an estimate of the missing transverse momentum $E_{\mathrm{T}}^{\text {miss }}$ resolution due to detector noise. To estimate this resolution on an event-by-event basis, minimum bias collision events without any real $E_{\mathrm{T}}^{\text {miss }}$ are analyzed and the resolution can be parameterized as a function of the scalar sum of the transverse momentum $\sum E_{\mathrm{T}}$. The expression used is $\sigma=a+b \sqrt{\sum E_{\mathrm{T}}}$, and the constants $a$ and $b$ are determined by fitting the resolution in slices of $\sqrt{\sum E_{\mathrm{T}}}$. Figure 1 shows the dependence of XS as a function of the number of pile-up collisions $\mu$ : in contrast to $E_{\mathrm{T}}^{\text {miss }}$, the pile-up dependence is much reduced which helps to keep trigger thresholds low. To make the XS trigger fully efficient, it is combined with a $E_{\mathrm{T}}^{\text {miss }}$ criterion. 

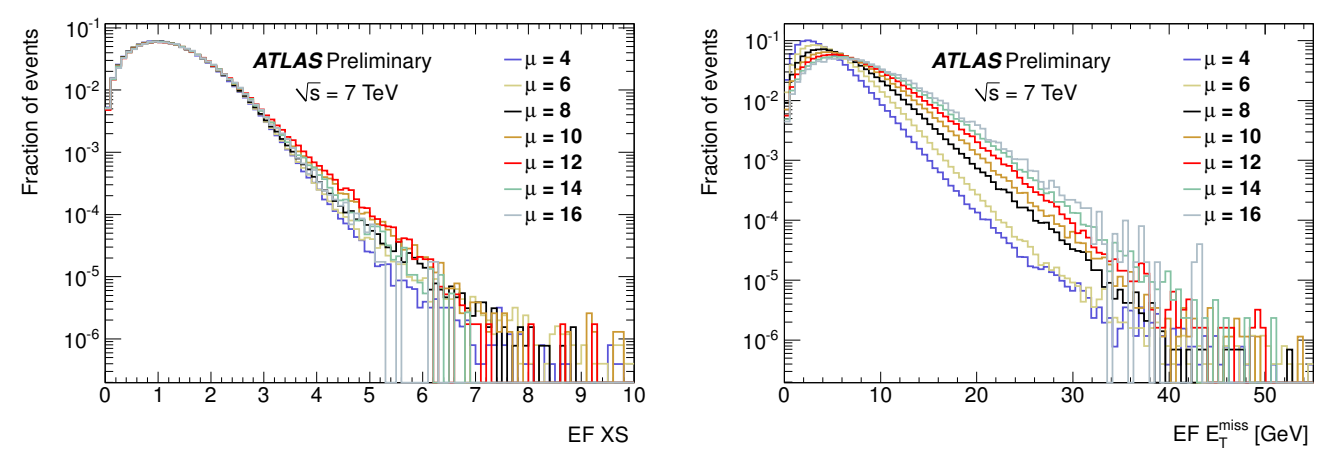

Figure 1: Right: the distribution of Event Filter (EF) $E_{\mathrm{T}}^{\text {miss }}$ for different values of pile-up collisions $\mu$. Left: the distribution of EF XS for different values of $\mu$. The comparison shows that the pile-up dependence is much reduced by using EF XS instead of $\mathrm{EF} E_{\mathrm{T}}^{\text {miss }}$. [5]

\section{New developments for Run II and first 2015 data}

The increased centre-of-mass energy and higher instantaneous luminosity create a challenging environment for the missing transverse momentum trigger. To keep the trigger thresholds low for physics, several upgrades on hardware, firmware and software side have been implemented. The expected number of coinciding pile-up collisions is about 55-80 per bunch crossing, which will significantly deteriorate the missing transverse momentum trigger resolution and would result in very high rates. To face these challenging conditions several new algorithms have been prepared during the shutdown period:

A new Kalman filter based algorithm at L1 is implemented to subtract pile-up energies and to apply a hadronic energy calibration. The resulting missing transverse momentum value has a better correlation with the actual hard scatter transverse momentum. The correction value is calculated from a weighted sum of L1 jets built from RoIs. The weights are optimized using a Kalman filter trained using monte carlo events with sizeable pile-up. The resulting correction weights $w_{i}$ are stored in a look-up table depending on the $\mathrm{L} 1$ jet $p_{\mathrm{T}}$ and $|\eta|$ :

$$
\vec{E}_{\mathrm{T} \mathrm{KF}}^{\mathrm{miss}}:=\vec{E}_{\mathrm{T} \mathrm{L} 1}^{\mathrm{miss}}-\sum w_{i} \times \vec{p}_{\mathrm{T}, \mathrm{i}-\mathrm{th}} \text { jet }
$$

In Fig. 2 the improvement in performance over the default L1 algorithms is shown, using a L1 threshold that corresponds to equal background rates.

For the HLT three new algorithms in addition to the cluster based algorithm from 2012 were implemented. Due to the merging of Level 2 and Event Filter more computing time can be spent to evaluate each event. The new algorithms are:

1. topoclPS : a cluster algorithm with hadronic calibration which subtracts average $\eta$ - dependent pile-up energy per unit area.

2. topoclPUC : cluster algorithm with hadronic calibration that requires at least one jet and performs a fit in which the total pile-up $E_{\mathrm{T}}^{\text {miss }}$ is constrained to zero. 


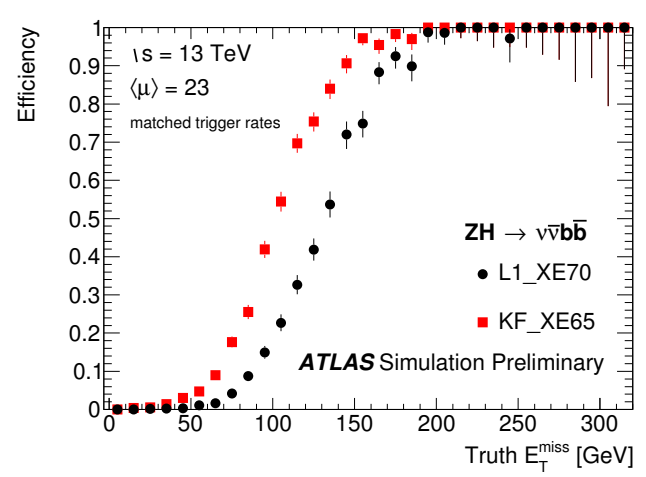

Figure 2: Trigger efficiency of L1 transverse missing momentum trigger and the Kalman filter KF-XE respectively for a simulated $\mathrm{ZH} \rightarrow v v b \bar{b}$ signal sample [5].

3. mht : A jet energy sum algorithm that uses the clustered jets : $H_{\mathrm{T}}^{\text {miss }}$.

In Figure 3 the missing transverse momentum distributions for all HLT algorithms are compared, using $78.7 \mathrm{pb}^{-1}$ of data recorded by ATLAS with $50 \mathrm{~ns}$ bunch spacing and a centre-of-mass energy of $\sqrt{s}=13 \mathrm{TeV}$.

\section{Conclusions}

The constant improvement throughout Run I led to reliable $E_{\mathrm{T}}^{\text {miss }}$ triggers for the ATLAS experiment. For Run II the change in centre-of-mass energy and instantaneous luminosity made the development of better trigger algorithms necessary in order to keep missing transverse momentum thresholds low for physics analyses. Level 1 performance shows a considerable improvement due to the new Kalman Filter based algorithm. The first performance plots of the $E_{\mathrm{T}}^{\text {miss }}$ triggers in 2015 data were shown. With the new development of intelligent trigger algorithms which reduce the dependency on pile-up both on Level 1 and HLT level, the ATLAS trigger is well prepared to face the challenges of high pile-up conditions in Run II.

\section{References}

[1] ATLAS Collaboration, The ATLAS Experiment at the CERN Large Hadron Collider, JINST 3 S08003 (2008).

[2] ATLAS Collaboration, Tech. Rep. CERN-LHCC-2003-022 ATLAS-TDR-16 (CERN, Geneva, 2003).

[3] ATLAS Collaboration, The ATLAS transverse-momentum trigger performance at the LHC in 2011, ATLAS-CONF-2014-002.

[4] ATLAS Collaboration, Tech. Rep. CERN-LHCC-2013-018. ATLAS-TDR-023 (CERN, Geneva, 2013).

[5] https://twiki.cern.ch/twiki/bin/view/AtlasPublic/MissingEtTriggerPublicResults 

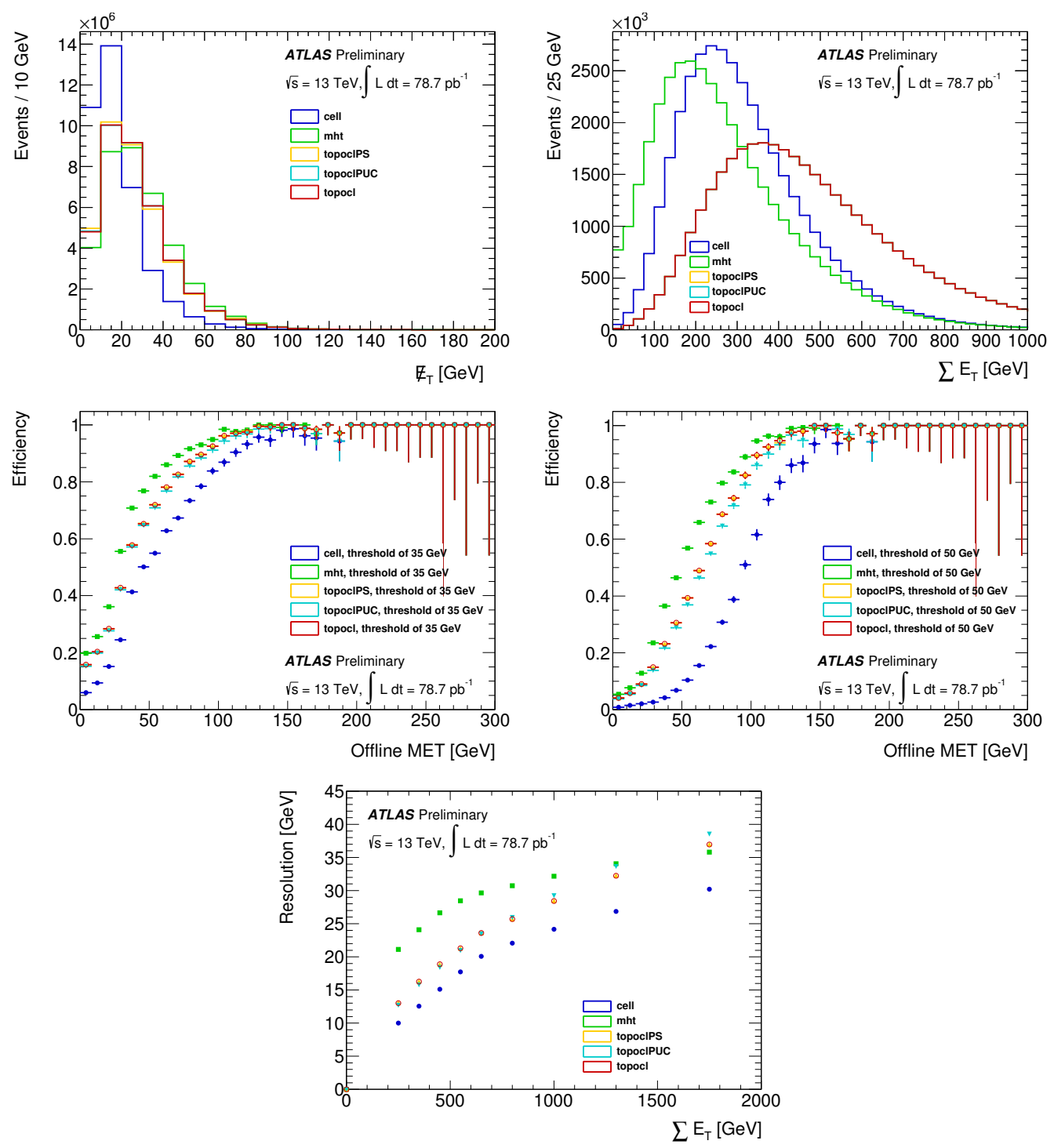

Figure 3: The transverse missing momentum performance for events taken with $\sqrt{s}=13 \mathrm{TeV}$ in 2015. Shown are comparisons of online $E_{\mathrm{T}}^{\text {miss }}$ and $\sum \mathrm{E}_{\mathrm{T}}$ distributions (top), the trigger efficiency for offline reconstructed $E_{\mathrm{T}}^{\text {miss }}$ (offline MET) for thresholds of $35 \mathrm{GeV}, 50 \mathrm{GeV}$ (middle) and the resolution in online $\sum \mathrm{E}_{\mathrm{T}}$ (bottom) for the following online algorithms: a 2-sided 2-sigma noise suppression cell-based algorithm (cell), a topocluster-based algorithm with no further corrections (topocl), an eta-ring pile-up subtraction (topoclPS), or a pile-up fit procedure (topoclPUC), and an algorithm based on the sum of jet momenta (mht). The three different topocluster-based algorithms overlay each other for most bins [5]. 